P91 (continued)

survey respondents believed food waste is a problem for most households, with big portion sizes the most often answered (30\%) reason for food waste. Respondents estimated $80 \%$ of food waste is thrown away, with leftovers more than twice as likely than fruits and vegetables to be thrown away. Only $12 \%$ correctly estimated the monetary value of food waste, while $80 \%$ underestimated the national average. Finally, 71\% of respondents were interested in decreasing food waste.

Conclusion: Findings show a need for providing online knowledge, tools and skills for food sustainability, which may influence WIC participant behaviors surrounding healthy food supplies. Practical applications include incorporating food sustainability concepts and behavior into WIC nutrition education, especially participants using wichealth.org, as a well as further research.

Funding: None.

\section{P92 Using the PSE Readiness Assessment and Decision Instrument to Support Implementation of Community Nutrition Strategies in Ohio}

Darcy Freedman,PhD, MPH, daf96@case.edu, Case Western Reserve University, 10900 Euclid Ave, Cleveland, $\mathrm{OH}$ 44106; Jennifer King, PhD, MS, Case Western Reserve University; Keren Valerus, MPH, MS, Case Western Reserve University; David Ngendahimana, PhD, Case Western Reserve University; Ana Claudia Zubieta, PhD, Ohio State University; Pat Bebo, MS, RD, LD, Ohio State University; Elizabeth Benninger, PhD, Case Western Reserve University; Carol Smathers, MPH, MS, Ohio State University

Objective: Promote practitioners' confidence to use and disseminate the PSE Readiness Assessment and Decision Instrument (PSE READI) to tailor PSE strategies to local contexts.

Use of Theory or Research: There is increasing attention within the Supplemental Nutrition Assistance Program-Education (SNAP-Ed) toward implementation of PSE strategies to improve dietary outcomes among lowincome populations. Tools to adapt PSEs to local levels of readiness and capacity are needed.

Target Audience: SNAP-Ed and public health practitioners in Ohio.

Program Description: The PSE READI was designed to adapt implementation of four community nutrition PSEs: farmers' markets, farm-to-school, healthy eating in childcare, healthy food retail. After completing the assessment, a tailored report is provided with three recommendations to guide local PSE implementation, which are linked to a resource library with $>1,000$ tools/guides. The tool was tested for usability and first disseminated via a day-long interactive training focused on supporting others use of the tool to assess, plan, and act to optimize PSE implementation in targeted areas.

Evaluation Methods: Pre- and post-survey design without randomization was used to test confidence in practitioners' ability to support and train others to use the PSE READI.
Results: Practitioners $(\mathrm{N}=23)$ doing work in 60 Ohio counties participated in the PSE READI training. One-third reported PSE implementation is related to their current work plan; 41\% supported others conducting PSEs. Confidence to support colleagues' implementation of PSEs (extremely/very confident: pre-35\%, post-70\%) and train colleagues to use the PSE READI tool (pre-10\%, Post-70\%) increased substantially after the training. The majority (87\%) planned to use the PSE READI tool in the next three months. Post-training, practitioners reported they were "better equipped," "more informed," and "inspired."

Conclusions: The PSE READI tool provides a feasible and efficient method to tailor PSE implementation to the realities of diverse communities. The tool and training were well-received by SNAP-Ed and public health practitioners resulting in an increase in practitioner confidence to support implementation of community nutrition PSEs.

Funding: USDA.

\section{P93 Utilizing Sensory Evaluation Methodology in Recipe Development for Expanded Food and Nutrition Education Program (EFNEP)}

Ginnefer Cox, PhD, RD, gocox@uga.edu, University of Georgia, 305 Sanford Dr, 176 Dawson Hall, Athens, GA 30602; Carla Moore, PhD, RDN, University of Georgia

Background: Federal programs such as EFNEP provide nutrition education for limited resource consumers. A component of the nutrition education often includes the inclusion of recipes which are provided to increase healthy eating behaviors among targeted audiences. Currently, there is limited information regarding sensory evaluation of recipes targeted to limited resource consumers. Objective: To utilize sensory evaluation methodology to assess acceptability including overall liking (OL) and liking of specific sensory attributes (appearance, flavor, texture) for 16 recipes taught and demonstrated through the University of Georgia EFNEP Food Talk nutrition education curriculum.

Study Design, Settings, Participants: A total of 872 EFNEP eligible Georgian adults who participate in the Food Talk Nutrition Education Curriculum conducted sensory evaluation on sixteen existing recipes through the EFNEP Food Talk curriculum. Recipes were prepared following standardized instructions for consistency of preparation and portion size.

Measurable Outcome/Analysis: Sensory evaluation used the traditional nine-point hedonic scale to evaluate OL and specified sensory attributes ( $1=$ dislike extremely, $5=$ neither like nor dislike, and $9=$ like extremely). Standard range of acceptability for a recipe is $\geq 7.0$. OL based on recipe title, and purchasing intent of recipe ingredients were also measured.

Results: Recipes with the highest acceptability ratings were fruit-based recipes (Fruit Smoothie, mean OL $=7.67$, flavor $=7.56$, texture $=7.54$; Crunchy Apple Salad, mean $\mathrm{OL}=7.97$, flavor $=8.18$, texture $=8.02$ ). Recipes with the lowest acceptability ratings were Easy Cheesy Broccoli Soup (mean $\mathrm{OL}=6.63$, flavor $=6.51$, texture $=6.64)$ and

Continued on page 575 